\title{
Topological Complexity of the Relative Closure of a Semi-Pfaffian Couple
}

\author{
Thierry Zell
}

Received: 31 March 2007 / Revised: 8 September 2007 /

Accepted: 3 January 2008 / Published online: 7 March 2008

(C) Springer Science+Business Media, LLC 2008

\begin{abstract}
Gabrielov introduced the notion of relative closure of a Pfaffian couple as an alternative construction of the o-minimal structure generated by Khovanskii's Pfaffian functions. In this paper, we use the notion of format (or complexity) of a Pfaffian couple to derive explicit upper bounds for the homology of its relative closure. We consider both the singular and the Borel-Moore homology theories.
\end{abstract}

Keywords Pfaffian functions $\cdot$ Fewnomials $\cdot$ o-Minimal structures $\cdot$ Betti numbers

\section{Introduction}

Pfaffian functions form a class of real-analytic functions with finiteness properties similar to that of polynomials (see Sect. 2.1). They were introduced by Khovanskii [16] who proved for them an analogue of the theorem of Bézout: a system of $n$ Pfaffian functions in $n$ variables can only have finitely many isolated solutions. Khovanskii's result suggests that any set defined using such well-behaved functions must be topologically tame, an idea which sparked interest in the construction of o-minimal structures containing the graphs of Pfaffian functions. (For the link between tame topology and o-minimality, and for the fundamental results of the theory, we refer the reader to [4] and [23].)

The first such structure was constructed by Wilkie [24] based on an idea of Charbonnel (the construction was generalized by Karpinski and Macintyre [15]). Drawing on the work of Lion and Rolin [17], Speissegger [22] built a larger structure that contains not only Khovanskii's Pfaffian functions but also every Rolle leaf of definable 1-forms. This structure is called the Pfaffian closure of the real field, and Lion and

T. Zell $(\bowtie)$

Department of Mathematics, Vassar College, 124 Raymond Avenue, Poughkeepsie, NY 12604, USA

e-mail: thzell@vassar.edu 
Speissegger [18] proved that this structure is model-complete in the language of Rolle leaves.

Complexity considerations led to a third approach by Gabrielov [8], limit sets. Khovanskii's upper bound on the number of isolated solutions can be expressed in terms of a tuple of integers called format. This format was used for many quantitative results on semi-Pfaffian sets (the sets defined by quantifier-free Pfaffian formulas), but did not seem adapted to Wilkie's construction. The structure obtained by Gabrielov is identical to Wilkie's, but the construction is entirely different, relying on the notion of relative closure $(X, Y)_{0}$ of a semi-Pfaffian couple $(X, Y)$ (see Sect. 2.3). Limit sets, which are finite unions of relative closures, coincide with the definable sets in Wilkie's structure. Highlighting the difference in approach, the main result of [24] is a theorem for complements, whereas the main theorem in [8] is for projections.

The relative closure $(X, Y)_{0}$ is the difference of the Hausdorff limits of two semiPfaffian families $X$ and $Y$ depending on one parameter $\lambda$. Since the fibers $X_{\lambda}$ and $Y_{\lambda}$ of the couple $(X, Y)$ are semi-Pfaffian, this lets us extend the notion of format to $(X, Y)_{0}$ by taking the component-wise maximum of the formats of $X_{\lambda}$ and $Y_{\lambda}$ (for some fixed small $\lambda>0$ ). This generalization of the notion of format allowed us to prove new quantitative results, such as upper bounds on the number of connected components [13] of a relative closure, and on the higher Betti numbers [26] under the assumption $Y=\varnothing$. In this paper, we conclude this study of the Betti numbers of relative closures by dealing with the case where $Y$ is not empty. Our results appear in Theorem 3.3 and 4.4, and they can be summarized in the following statement.

Main Result Let $H_{*}$ and $H_{*}^{\mathrm{BM}}$ denote the homology groups associated, respectively, to the singular and the Borel-Moore theories. Then, for any semi-Pfaffian couple $(X, Y)$, the ranks of the groups

$$
H_{k}\left((X, Y)_{0}\right) \text { and } H_{k}^{\mathrm{BM}}\left((X, Y)_{0}\right)
$$

admit an upper bound that is an explicit function of $k$ and of the format of the couple $(X, Y)$. In particular, the dependence in the parameter variable $\lambda$ of the format of the semi-Pfaffian sets $X$ and $Y$ is not involved in these estimates.

We leave the detailed definitions and specific estimates for later sections. The Borel-Moore case (Theorem 3.3) is a reduction to the case $Y=\varnothing$. The singular case (Theorem 4.4) is more involved: it features a reduction to a definable Hausdorff limit of a family defined by a quantified Pfaffian formula. Following [26], the Betti numbers of that limit can be bounded by the Betti numbers of simpler sets, but since these simpler sets are also defined by quantified formulas, they require an extra use of the descent spectral sequence before the complexity bounds can finally be established.

The paper is organized as follows: Sect. 2 introduces the Pfaffian structure and Gabrielov's construction of it via relative closures. It also presents all the spectral sequence machinery and its corollaries that appeared first in [14] and [26], and which will be used in our proofs. Section 3 is devoted to the Borel-Moore estimates, and Sect. 4 to the singular case.

Notations For any $\mathbf{x} \in \mathbb{R}^{n},|\mathbf{x}|$ denotes the Euclidean norm of $\mathbf{x}$. For any set $X \subseteq \mathbb{R}^{n}$, $\bar{X}$ denotes the closure of $X$ in the Euclidean topology, and $\partial X=\bar{X} \backslash X$ denotes the 
frontier of $X$. The homology $H(X)$ considered is the singular homology with coefficients in a fixed group $G$ (which is omitted, as the choice of $G$ does not affect the results). The $k$ th Betti number of $X$ is defined as the rank of the homology group $H_{k}(X)$. We denote it by $b_{k}(X)$ and let $b(X)=\sum_{k} b_{k}(X)$.

\section{Preliminaries}

In this section, we discuss Pfaffian functions and related notions: semi-Pfaffian sets, sub-Pfaffian sets, relative closures and limit sets. To each of these constructions, we associate a notion of complexity that we call format. The reader can find more details on Pfaffian sets and complexity results in the survey [11].

\subsection{Pfaffian Functions}

Let $\mathcal{U} \subseteq \mathbb{R}^{n}$ be an open domain. The following definition is due to Khovanskii [16].

Definition 2.1 Let $\mathbf{x}=\left(x_{1}, \ldots, x_{n}\right)$ and let $\left(f_{1}(\mathbf{x}), \ldots, f_{\ell}(\mathbf{x})\right)$ be a sequence of analytic functions in $\mathcal{U}$. This sequence is called a Pfaffian chain if the functions $f_{i}$ are solution on $\mathcal{U}$ of a triangular differential system of the form

$$
d f_{i}(\mathbf{x})=\sum_{j=1}^{n} P_{i, j}\left(\mathbf{x}, f_{1}(\mathbf{x}), \ldots, f_{i}(\mathbf{x})\right) d x_{j}
$$

where the functions $P_{i, j}$ are polynomials in $\mathbf{x}$ and $\left(f_{1}, \ldots, f_{i}\right)$.

Definition 2.2 Let $\left(f_{1}, \ldots, f_{\ell}\right)$ be a fixed Pfaffian chain on a domain $\mathcal{U}$. The function $q$ is a Pfaffian function expressible in the chain $\left(f_{1}, \ldots, f_{\ell}\right)$ if there exists a polynomial $Q$ such that for all $\mathbf{x} \in \mathcal{U}$,

$$
q(\mathbf{x})=Q\left(\mathbf{x}, f_{1}(\mathbf{x}), \ldots, f_{\ell}(\mathbf{x})\right)
$$

In general, a function $q: \mathcal{U} \rightarrow \mathbb{R}$ is called Pfaffian if it is expressible in some Pfaffian chain $\left(f_{1}, \ldots, f_{\ell}\right)$ defined on $\mathcal{U}$.

If $\left(f_{1}, \ldots, f_{\ell}\right)$ is a Pfaffian chain, we call $\ell$ its length, and we let its degree $\alpha$ be the maximum of the degrees of the polynomials $P_{i, j}$ appearing in (1). If $q$ is as in (2), the degree $\beta$ of the polynomial $Q$ is called the degree of $q$ in the chain $\left(f_{1}, \ldots, f_{\ell}\right)$.

Definition 2.3 For $q$ as above, the tuple $(n, \ell, \alpha, \beta)$ is called the format of $q$.

Pfaffian functions form a large class that contains, among other things, real elementary functions and Liouvillian functions (see [16]). For more on the practical complexity of Pfaffian functions, the papers [9-11] contain examples of elementary functions, their various Pfaffian representations, and the associated formats. 


\subsection{Semi-Pfaffian Sets}

We fix a Pfaffian chain $\left(f_{1}, \ldots, f_{\ell}\right)$. We consider the restriction of these functions to a domain $\mathcal{U}$ of the form

$$
\mathcal{U}=\left\{\mathbf{x} \in \mathbb{R}^{n} \mid g_{1}(\mathbf{x})>0, \ldots, g_{k}(\mathbf{x})>0\right\},
$$

where $g_{1}, \ldots, g_{k}$ are Pfaffian functions that are expressible in the chain $\left(f_{1}, \ldots, f_{\ell}\right)$.

Definition 2.4 Let $\mathcal{P}=\left\{p_{1}, \ldots, p_{s}\right\}$ be a set of Pfaffian functions expressible in the chain $\left(f_{1}, \ldots, f_{\ell}\right)$. A formula $\Phi$ is called a quantifier-free Pfaffian formula on $\mathcal{P}$ if it is derived from atoms of the form $p_{i} \star 0$ for $1 \leq i \leq s$ and $\star \in\{=,<,>\}$, using conjunctions, disjunctions and negations.

Definition 2.5 A subset $X \subseteq \mathbb{R}^{n}$ is called a semi-Pfaffian set if there exists a quantifier-free Pfaffian formula $\Phi$ whose atoms are Pfaffian functions expressible in some chain $\left(f_{1}, \ldots, f_{\ell}\right)$ defined on a domain $\mathcal{U} \subseteq \mathbb{R}^{n}$ of the form (3) such that $X=\{\mathbf{x} \in \mathcal{U} \mid \Phi(\mathbf{x})\}$.

The semi-Pfaffian set $X$ is called restricted if and only if $\bar{X} \subseteq \mathcal{U}$.

Remark 2.6 If $X$ is a restricted semi-Pfaffian set, its closure is also semi-Pfaffian [7], a fact which is not true for nonrestricted sets [9]. This fact will be used in Sect. 3.2.

Definition 2.7 Let $\left(f_{1}, \ldots, f_{\ell}\right)$ be a fixed Pfaffian chain and $\mathcal{P}=\left\{p_{1}, \ldots, p_{s}\right\}$ be a collection of $s$ Pfaffian functions expressible in that chain. If the format of each $p_{i}$ is bounded by $(n, \ell, \alpha, \beta)$, then the format of any quantifier-free Pfaffian formula on $\mathcal{P}$, as well as the format of the corresponding semi-Pfaffian set, is $(n, \ell, \alpha, \beta, s)$.

Khovanskii's estimate on the number of solutions of a system of Pfaffian equations [16] allows, using arguments from Morse theory, to bound the Betti numbers of any set which is defined as the common zeros of a family of Pfaffian functions $[16,25]$. This is used to derive the following bound for semi-Pfaffian sets.

Theorem 2.8 Let $X$ be any semi-Pfaffian set defined by a quantifier-free Pfaffian formula of format $(n, \ell, \alpha, \beta, s)$. The sum of the Betti numbers of $X$ admits a bound of the form

$$
b(X) \leq 2^{\ell(\ell-1) / 2} s^{2 n} O(n(\alpha+\beta))^{n+\ell},
$$

where the constant coming from the $O$ notation depends only on the definable domain $\mathcal{U}$.

The estimate (4) follows from the deformation techniques used in [12] in the algebraic setting, by applying the bound (for so-called $\mathcal{P}$-closed formulas as introduced in [1]) appearing in [25]. 


\subsection{Relative Closure and Limit Sets}

Projections of semi-Pfaffian sets may not always be semi-Pfaffian [9, 20], but Wilkie showed in [24] that Pfaffian functions nonetheless generate an o-minimal structure. We refer the reader to [4] and [23] for a detailed account of the fundamental properties of o-minimal structures.

In [8], Gabrielov obtained an o-minimal structure containing the graphs of Pfaffian functions and in which any definable set can be given a format. The construction involves the notion of relative closure. We will denote the structure obtained in [8] by $\mathcal{S}$.

To obtain all $\mathcal{S}$-definable sets in $\mathbb{R}^{n}$, we need to consider semi-Pfaffian sets defined in a domain $\mathcal{U} \subseteq \mathbb{R}^{n} \times \mathbb{R}_{+}$. Without loss of generality, we will assume that these sets are bounded (see Remark 2.15). We write $\mathbf{x}=\left(x_{1}, \ldots, x_{n}\right)$ for the coordinates in $\mathbb{R}^{n}$ and $\lambda$ for the last coordinate (which we think of as a parameter). If $X$ is a subset of $\mathcal{U}$ and $\lambda>0$, the fiber $X_{\lambda}$ is defined by

$$
X_{\lambda}=\{\mathbf{x} \mid(\mathbf{x}, \lambda) \in X\} \subseteq \mathbb{R}^{n},
$$

and we consider $X$ as the family of its fibers $X_{\lambda}$. We let $X_{+}=X \cap\{\lambda>0\}$ and denote by $\check{X}$ the Hausdorff limit of the family $\overline{X_{\lambda}}$ as $\lambda$ goes to zero;

$$
\check{X}=\left\{\mathbf{x} \in \mathbb{R}^{n} \mid(\mathbf{x}, 0) \in \overline{X_{+}}\right\} .
$$

Definition 2.9 Let $X$ be a semi-Pfaffian subset of $\mathcal{U}$. The set $X$ constitutes a semiPfaffian family if for any $\varepsilon>0$, the set $X \cap\{\lambda>\varepsilon\}$ is restricted. (Recall that by definition, the set $X \cap\{\lambda>\varepsilon\}$ is restricted if its topological closure is contained in $\mathcal{U}$.)

Definition 2.10 Let $X$ and $Y$ be semi-Pfaffian families in $\mathcal{U}$ defined in a common chain $\left(f_{1}, \ldots, f_{\ell}\right)$. They form a semi-Pfaffian couple if and only if, for all $\lambda>0$, we have $\overline{Y_{\lambda}}=Y_{\lambda}$ and $\partial\left(X_{\lambda}\right) \subseteq Y_{\lambda}$.

Definition 2.11 The format $(n, \ell, \alpha, \beta, s)$ of a semi-Pfaffian family $X$ is the format of the fiber $X_{\lambda}$ for a small $\lambda>0$. Then, the format of the couple $(X, Y)$ is the component-wise maximum of the format of the families $X$ and $Y{ }^{1}$

Definition 2.12 Let $(X, Y)$ be a semi-Pfaffian couple in $\mathcal{U}$. We define the relative closure of $(X, Y)$ at $\lambda=0$ by

$$
(X, Y)_{0}=\check{X} \backslash \check{Y} \subseteq \check{\mathcal{U}}
$$

where $\check{X}, \check{Y}$ and $\check{\mathcal{U}}$ denote the Hausdorff limits of the respective fibers as in (5).

Remark 2.13 The restrictions on semi-Pfaffian couples (Definition 2.10) imply that for $(X, \varnothing)$ to be a couple, we must have $\partial\left(X_{\lambda}\right)=\varnothing$ for all $\lambda>0$, and since $X$ is

\footnotetext{
${ }^{1}$ Note that the format of $X$ as a semi-Pfaffian set is different from its format as a semi-Pfaffian family, since $X$ has $n+1$ variables as a set but only $n$ as a family. 
bounded, $X_{\lambda}$ must be compact. We will denote by $X_{0}$ the relative closure $(X, \varnothing)_{0}$. In that case, $X_{0}$ is simply the Hausdorff limit of the family of compacts $X_{\lambda}$ when $\lambda$ goes to zero.

Definition 2.14 Let $\Omega \subseteq \mathbb{R}^{n}$ be an open, semi-Pfaffian domain. A limit set $Z \subseteq$ $\Omega$ is a set of the form $Z=\left(X_{1}, Y_{1}\right)_{0} \cup \cdots \cup\left(X_{k}, Y_{k}\right)_{0}$, where $\left(X_{i}, Y_{i}\right)$ are semiPfaffian couples, respectively defined in domains $\mathcal{U}_{i} \subseteq \mathbb{R}^{n} \times \mathbb{R}_{+}$, such that $\check{\mathcal{U}}_{i}=\bar{\Omega}$ for $1 \leq i \leq k$. If the formats of the couples $\left(X_{i}, Y_{i}\right)$ are bounded component-wise by $(n, \ell, \alpha, \beta, s)$ we say that the format of the limit set is $(n, \ell, \alpha, \beta, s, k)$

The main result of [8] is that limit sets are exactly the definable sets in $\mathcal{S}$. Moreover, the notion of format for limit sets makes the structure effective, to the extent that when performing Boolean operations on limit sets, the resulting formats can be explicitly bounded in terms of the formats of the original sets.

Remark 2.15 When defining semi-Pfaffian couples, we assume, as in [8], that the semi-Pfaffian families $X$ and $Y$ are bounded. This restriction allows us to avoid a separate treatment of infinity: we can see $\mathbb{R}^{n}$ as embedded in $\mathbb{R} \mathbb{P}^{n}$, in which case any set we consider can be subdivided into pieces that are relatively compact in their own charts.

\subsection{Betti Numbers of Sub-Pfaffian Sets and of Hausdorff Limits}

The first upper bounds for the Betti numbers of Pfaffian sets which were not defined by quantifier-free formulas were obtained in [14] (for sub-Pfaffian sets) and in [26] (for Hausdorff limits). These results will be the key to our estimates for relative closures. Both results follow from the descent inequality presented in Theorem 2.18. First, let's recall the following definition.

Definition 2.16 Let $X$ and $Y$ be two topological spaces, and let $f: X \rightarrow Y$ be a continuous surjection. The map $f$ is called locally split if for any $y \in Y$, there exists a continuous section of $f$ defined in a neighborhood of $y$.

The following lemma gives a wide class of locally split maps.

Lemma 2.17 Let $A$ and $B$ be topological spaces. If $U \subseteq A \times B$ is an open subset for the product topology, then the restriction to $U$ of the standard projection $\Pi: A \times B \rightarrow A$ is locally split.

The proof of Lemma 2.17 is elementary and we will omit it here. The descent inequality is the following theorem.

Theorem 2.18 Let $f: X \rightarrow Y$ be a continuous surjective map definable in an o-minimal structure. Let $W_{f}^{p}(X)$ be the $(p+1)$-fold fibered product of $X$,

$$
W_{f}^{p}(X)=\left\{\left(\mathbf{x}_{0}, \ldots, \mathbf{x}_{p}\right) \in X^{p+1} \mid f\left(\mathbf{x}_{0}\right)=\cdots=f\left(\mathbf{x}_{p}\right)\right\} .
$$


Suppose that $f$ is either closed or locally split. Then, for all $k$, the following inequality holds:

$$
b_{k}(Y) \leq \sum_{p+q=k} b_{q}\left(W_{f}^{p}(X)\right) .
$$

The inequality (8) follows from the existence of a spectral sequence $E_{p, q}^{r}$ which converges to the homology of $Y$, and such that $E_{p, q}^{1} \cong H_{q}\left(W_{f}^{p}(X)\right)$. The sequence $E_{p, q}^{r}$ is sometimes known as the homological descent spectral sequence. It seems it first appeared in [5] in the case of proper maps, and it has been rediscovered many times since. The reader can find proofs in [14] for the closed case and in [2] or [6] for the locally split case.

Remark 2.19 Note that an assumption on $f$ such as closed or locally split is necessary in Theorem 2.18 (see [26]).

Definition 2.20 A set $Y \subseteq \mathbb{R}^{n}$ is called sub-Pfaffian if there exists an integer $r$ and a semi-Pfaffian set $X \subseteq \mathbb{R}^{n+r}$ such that $Y=\Pi(X)$, where $\Pi$ is the standard projection $\mathbb{R}^{n+r} \rightarrow \mathbb{R}^{n}$.

When the inequality (8) holds for such a projection $\Pi$, we can apply Theorem 2.8 and obtain the following estimate on the Betti numbers of the resulting sub-Pfaffian set (see [14]).

Corollary 2.21 Let $X \subseteq \mathbb{R}^{n+r}$ be a semi-Pfaffian set of format $(n+r, \ell, \alpha, \beta, s)$. Denote by $\Pi$ the standard projection $\mathbb{R}^{n+r} \rightarrow \mathbb{R}^{n}$ and let $Y=\Pi(X)$. Assume that the restriction $\left.\Pi\right|_{X}$ is either closed or locally split. Then, we have for all $k \geq 1$,

$$
b_{k-1}(Y) \leq 2^{k \ell(k \ell-1) / 2} s^{2(n+k r)} O((n+k r)(\alpha+\beta))^{n+k(r+\ell)} .
$$

Another corollary of Theorem 2.18 is the following.

Theorem 2.22 ([26, Theorem 1]) Let $A \subseteq \mathbb{R}^{n+r}$ be a bounded set definable in some $o$-minimal structure and let $A^{\prime}$ be its projection to $\mathbb{R}^{r}$. Suppose that the fibers $A_{a} \subseteq$ $\mathbb{R}^{n}$ are compact for all values of the parameter $a \in A^{\prime}$, and let $L$ be the Hausdorff limit of some sequence of fibers $\left(A_{a_{i}}\right)$. Then, there exists definable sets $D_{a}^{p}(\varepsilon)$, where $a \in A^{\prime}$ and $\varepsilon>0$. such that for any integer $k$, we have

$$
b_{k}(L) \leq \sum_{p+q=k} b_{q}\left(D_{a}^{p}(\varepsilon)\right)
$$

The set $D_{a}^{p}(\varepsilon)$ is called the expanded $p$ th diagonal of the fiber $A_{a}$, and is defined by

$$
D_{a}^{p}(\varepsilon)=\left\{\left(\mathbf{x}_{0}, \ldots, \mathbf{x}_{p}\right) \in\left(A_{a}\right)^{p+1} \mid \rho_{p}\left(\mathbf{x}_{0}, \ldots, \mathbf{x}_{p}\right) \leq \varepsilon\right\},
$$

where $\rho_{0}=0$ and $\rho_{p}\left(\mathbf{x}_{0}, \ldots, \mathbf{x}_{p}\right)=\sum_{0 \leq i<j \leq p}\left|\mathbf{x}_{i}-\mathbf{x}_{j}\right|^{2}$ for $p>0$. 


\section{Borel-Moore Homology of Relative Closures}

In this section, we estimate the rank of the Borel-Moore homology groups of the relative closure of a Pfaffian couple, in terms of the format of the couple. Upper bounds on the ranks of the Borel-Moore groups can be used to establish lower bounds in computational complexity; see, for instance, [19] for a treatment of the semialgebraic case.

\subsection{Borel-Moore Homology in o-Minimal Structures}

In the o-minimal setting, the Borel-Moore homology of a locally closed, definable set has a simple description given in the following definition. We refer the reader to [3, Sect. 11.7] for more details.

Definition 3.1 Let $S \subseteq \mathbb{R}^{n}$ be a set definable in some o-minimal structure. If $S$ is compact, the Borel-Moore homology is simply $H_{*}^{\mathrm{BM}}(S)=H_{*}(S)$. If $S=A \backslash B$ for some definable compact sets $A$ and $B$ such that $B \subseteq A$, the Borel-Moore homology $H_{*}^{\mathrm{BM}}(S)$ is

$$
H_{*}^{\mathrm{BM}}(S)=H_{*}(A, B) .
$$

Note that the Borel-Moore homology groups are not defined for all definable subsets, but only for locally closed sets, i.e. sets that can be written in the form $U \cap F$ where $U$ is open and $F$ is closed.

We will denote by $b_{k}^{\mathrm{BM}}(S)$ the rank of the group $H_{k}^{\mathrm{BM}}(S)$. The Borel-Moore theory has an attractive property: the numbers $b_{k}^{\mathrm{BM}}$ are subadditive.

Proposition 3.2 ([3, Proposition 11.7.5]) Let $S$ be a locally closed definable set and $T \subseteq S$ a closed definable subset of $S$. Then, there exists a long exact sequence

$$
\cdots \longrightarrow H_{k}^{\mathrm{BM}}(T) \longrightarrow H_{k}^{\mathrm{BM}}(S) \longrightarrow H_{k}^{\mathrm{BM}}(S \backslash T) \longrightarrow H_{k-1}^{\mathrm{BM}}(T) \longrightarrow \cdots
$$

In particular, the following inequality holds for all integer $k$ :

$$
b_{k}^{\mathrm{BM}}(S \backslash T) \leq b_{k}^{\mathrm{BM}}(S)+b_{k-1}^{\mathrm{BM}}(T) .
$$

This subadditivity property is the key to bounding Borel-Moore ranks of relative closures.

\subsection{Effective Estimates in the Pfaffian Structure}

Let us consider now a semi-Pfaffian couple $(X, Y)$. For now, we will assume that the fibers $X_{\lambda}$ and $Y_{\lambda}$ are compact for all $\lambda>0$, and that their Hausdorff limits $\check{X}$ and $\check{Y}$ verify $\check{Y} \subseteq \check{X}$. Under these hypotheses, we have the following theorem.

Theorem 3.3 Let $(X, Y)$ be a semi-Pfaffian couple as above. If the format of the couple $(X, Y)$ is bounded by $(n, \ell, \alpha, \beta, s)$ then, for any integer $k \geq 1$, we have

$$
b_{k-1}^{\mathrm{BM}}\left((X, Y)_{0}\right) \leq 2^{k \ell(k \ell-1) / 2} s^{2 n k} O(k n(\alpha+\beta))^{k(n+\ell)} .
$$


Proof By definition we have $(X, Y)_{0}=\check{X} \backslash \check{Y}$. Since $\check{X}$ and $\check{Y}$ are compact sets such that $\check{Y} \subseteq \check{X}$, Proposition 3.2 gives

$$
b_{k-1}^{\mathrm{BM}}\left((X, Y)_{0}\right) \leq b_{k-1}^{\mathrm{BM}}(\check{X})+b_{k-2}^{\mathrm{BM}}(\check{Y}) .
$$

As the sets $\check{X}$ and $\check{Y}$ are compact, their Borel-Moore homology coincides with the singular one, so that the previous relation becomes

$$
b_{k-1}^{\mathrm{BM}}\left((X, Y)_{0}\right) \leq b_{k-1}(\check{X})+b_{k-2}(\check{Y}) .
$$

Since $\check{X}$ (resp. $\check{Y}$ ) is the Hausdorff limit of the family of compact sets $X_{\lambda}$ (resp. $Y_{\lambda}$ ) when $\lambda$ goes to zero, the ranks $b_{k-1}(\check{X})$ and $b_{k-2}(\check{Y})$ can be estimated using Theorem 2.22 (see [26] for more details). The bound obtained is (13).

For a general semi-Pfaffian couple $(X, Y)$, two things can go wrong in the previous argument: the fibers $X_{\lambda}$ may not closed, ${ }^{2}$ and we may not have $\check{Y} \subseteq \check{X}$. If $\check{Y} \nsubseteq \check{X}$, we can simply consider the couple $(X \cup Y, Y)$ which trivially verifies $(X \cup Y, Y)_{0}=(X, Y)_{0}$. The complexity of both couples is essentially the same, and the inequality (13) still holds.

If the fibers $X_{\lambda}$ are not compact, a (weaker) bound can still be established: since $X_{\lambda}$ is restricted, its closure $\overline{X_{\lambda}}$ is also semi-Pfaffian, and its complexity can be estimated using Theorem 1.1 of [7]. Since taking the closure does not change the Hausdorff limit $\check{X}$, we can apply the above theorem to the couple $(\bar{X}, Y)$. However, the format of $\bar{X}$ involves degrees that are doubly exponential in $n$, so the bound on $b_{k-1}^{\mathrm{BM}}\left((X, Y)_{0}\right)$ is much worse than (13).

\section{Singular Homology of Relative Closures}

We will now establish a single-exponential upper bound on the rank of the singular homology groups of Pfaffian relative closures. Given a semi-Pfaffian couple $(X, Y)$, we begin by constructing a family with compact fibers $K$ which is defined using a Pfaffian formula with a single universal quantifier, and whose Hausdorff limit has the same homology groups as $(X, Y)_{0}$.

\subsection{Reduction to Hausdorff Limits}

Let $(X, Y)$ be a semi-Pfaffian couple and $(X, Y)_{0}$ be its relative closure. If $\delta$ : $(0,1) \rightarrow \mathbb{R}_{+}$is any function definable in $\mathcal{S}$, we let

$$
\begin{aligned}
K & =\left\{(\mathbf{x}, \lambda) \in X \cap\{\lambda>0\} \mid \operatorname{dist}\left(\mathbf{x}, Y_{\lambda}\right) \geq \delta(\lambda)\right\} \\
& =\left\{(\mathbf{x}, \lambda) \in X \cap\{\lambda>0\}\left|\forall \mathbf{y} \in Y_{\lambda},\right| \mathbf{x}-\mathbf{y} \mid \geq \delta(\lambda)\right\} .
\end{aligned}
$$

\footnotetext{
${ }^{2}$ But remember that by definition of a semi-Pfaffian couple (Definition 2.10), the fibers $Y_{\lambda}$ have to be closed. 
The set $K$ is definable in $\mathcal{S}$; moreover, for any $\lambda>0$, the set $K_{\lambda}$ is compact. Indeed, the fibers $X_{\lambda}$ are bounded by assumption (see Remark 2.15) and $K_{\lambda}$ is a closed subset of $X_{\lambda}$, so in order for $K_{\lambda}$ to be noncompact, it would have to contain points in the frontier $\partial\left(X_{\lambda}\right)$. Equation (14) shows that $K_{\lambda} \cap Y_{\lambda}=\varnothing$, and as the definition of semiPfaffian couple (Definition 2.10) requires that $\partial\left(X_{\lambda}\right) \subseteq Y_{\lambda}$, the compactness of $K_{\lambda}$ follows.

Proposition 4.1 Let $(X, Y)$ be a semi-Pfaffian couple. Given a definable function $\delta(\lambda)$ as above and $K$ defined as in (14), denote by $\delta_{0}$ the limit of $\delta(\lambda)$ and by $K_{0}$ be the Hausdorff limit of the fibers $K_{\lambda}$ (both limits taken when $\lambda$ goes to zero). Then, there exists $\delta_{1}>0$ such that, for all $k \in \mathbb{N}$ and for all choice of $\delta(\lambda)$ such that $\delta_{0} \in\left(0, \delta_{1}\right)$, we have

$$
b_{k}\left((X, Y)_{0}\right)=b_{k}\left(K_{0}\right)
$$

Proof Let $K(\delta)$ be the definable subset given, for any $\delta>0$, by

$$
K(\delta)=\{\mathbf{x} \in \check{X} \mid \operatorname{dist}(\mathbf{x}, \check{Y}) \geq \delta\} .
$$

Since $(X, Y)_{0}=\{\mathbf{x} \in \check{X} \mid \operatorname{dist}(\mathbf{x}, \check{Y})>0\}$, the sets $K(\delta)$ are compact subset of $(X, Y)_{0}$ for all $\delta$ small enough. The singular chain complexes $\left\{C_{*}(K(\delta))\right\}$ form a directed system for the inclusion maps. A singular chain of $(X, Y)_{0}$ has a support which is contained in a set $K(\delta)$ for a small enough value of $\delta$; thus, the direct limit of the system $\left\{C_{*}(K(\delta))\right\}$ is $C_{*}\left((X, Y)_{0}\right)$. By Theorem 4.1.7 of [21], the homology and direct limit functors commute, giving a similar equality for the singular homology groups. In particular, we must have

$$
b_{k}\left((X, Y)_{0}\right)=\lim _{\delta \rightarrow 0} b_{k}(K(\delta)) .
$$

Since the family $K(\delta)$ is definable in $\mathcal{S}$, the generic triviality theorem (see Theorem 1.2 in Chap. 9 of [23] or Theorem 5.22 in [4]) guarantees that we can find some real number $\delta_{1}>0$ such that the topological type of the sets $K(\delta)$ is constant for $\delta \in\left(0, \delta_{1}\right)$. The relation (16) implies that

$$
\forall \delta \in\left(0, \delta_{1}\right), \quad b_{k}\left((X, Y)_{0}\right)=b_{k}(K(\delta)) .
$$

For the family $K$ defined in (14), the Hausdorff limit $K_{0}$ of the fibers $K_{\lambda}$ as $\lambda$ goes to zero is $K_{0}=K\left(\delta_{0}\right)$. Thus, any definable function $\delta(\lambda)$ that verifies $\delta_{0} \in\left(0, \delta_{1}\right)$ yields a family $K$ such that (15) holds.

Since $K_{0}$ is the Hausdorff limit of the definable family $K_{\lambda}$ when $\lambda$ goes to zero, we can use Theorem 2.22 to bound the Betti numbers of $K_{0}$. Thus, there exists $\lambda>0$ and $\varepsilon>0$ such that

$$
b_{k}\left(K_{0}\right) \leq \sum_{p+q=k} b_{q}\left(D_{\lambda}^{p}(\varepsilon)\right),
$$

where $D_{\lambda}^{p}(\varepsilon)$ denotes the expanded diagonals of $K_{\lambda}$,

$$
D_{\lambda}^{p}(\varepsilon)=\left\{\left(\mathbf{x}_{0}, \ldots, \mathbf{x}_{p}\right) \in\left(K_{\lambda}\right)^{p+1} \mid \rho_{p}\left(\mathbf{x}_{0}, \ldots, \mathbf{x}_{p}\right) \leq \varepsilon\right\} .
$$


The set $D_{\lambda}^{p}(\varepsilon)$ is defined by a Pfaffian formula which is the conjunction of a quantifier-free part

$$
\left(\mathbf{x}_{0}, \ldots, \mathbf{x}_{p}\right) \in\left(X_{\lambda}\right)^{p+1} \wedge \rho_{p}\left(\mathbf{x}_{0}, \ldots, \mathbf{x}_{p}\right) \leq \varepsilon
$$

and a part using a single universal quantifier,

$$
\forall \mathbf{y} \in Y_{\lambda}, \quad \bigwedge_{i=0}^{p}\left|\mathbf{x}_{i}-\mathbf{y}\right| \geq \delta(\lambda)
$$

\subsection{Complements and Duality}

Proposition 4.1 and inequality (17) reduce our problem to bounding the Betti numbers of the sets $D_{\lambda}^{p}(\varepsilon)$, or equivalently (via Alexander duality), of their complements. The sets $\left(\mathcal{U}_{\lambda}\right)^{p+1} \backslash D_{\lambda}^{p}(\varepsilon)$ are defined by existential Pfaffian formulas; our next result estimates their Betti numbers using the results from Sect. 2.4

Proposition 4.2 Let $(X, Y)$ be a semi-Pfaffian family of format bounded by $(n, \ell, \alpha, \beta, s)$ defined in a domain $\mathcal{U}$. Let $p$ be some fixed integer, $\lambda$ and $\varepsilon$ be positive real numbers, and let $D_{\lambda}^{p}(\varepsilon)$ be the set defined in (18). For any integer $q$, the Betti number $b_{q-1}\left(\left(\mathcal{U}_{\lambda}\right)^{p+1} \backslash D_{\lambda}^{p}(\varepsilon)\right)$ is bounded by

$$
2^{[q(p+2) \ell]^{2} / 2}[s(p+1)]^{O((p+q) n)}[n(p+q)(\alpha+\beta)]^{O((p+q)(n+\ell))} .
$$

Proof In order to simplify notations, we let $\Omega=\left(\mathcal{U}_{\lambda}\right)^{p+1}, D=D_{\lambda}^{p}(\varepsilon), \delta=\delta(\lambda)$, $\mathcal{X}=\left(X_{\lambda}\right)^{p+1}$ and $\mathcal{Y}=Y_{\lambda}$. With these notations, $D$ is the set of tuples $\left(\mathbf{x}_{0}, \ldots, \mathbf{x}_{p}\right)$ such that

$$
\left(\mathbf{x}_{0}, \ldots, \mathbf{x}_{p}\right) \in \mathcal{X} \wedge \rho_{p}\left(\mathbf{x}_{0}, \ldots, \mathbf{x}_{p}\right) \leq \varepsilon \wedge \forall \mathbf{y} \in \mathcal{Y}, \quad \bigwedge_{i=0}^{p}\left|\mathbf{x}_{i}-\mathbf{y}_{i}\right| \geq \delta
$$

Let $\Pi: \Omega \times \mathcal{Y} \rightarrow \Omega$ denote the projection on the first factor, and let $\mathcal{E}=A \cup$ $B \cup C$, where

$$
\begin{aligned}
& A=\bigcup_{i=0}^{p}\left\{\left(\mathbf{x}_{0}, \ldots, \mathbf{x}_{p}, \mathbf{y}\right) \in \Omega \times \mathcal{Y}|| \mathbf{x}_{i}-\mathbf{y} \mid<\delta\right\}, \\
& B=\left\{\left(\mathbf{x}_{0}, \ldots, \mathbf{x}_{p}, \mathbf{y}\right) \in \Omega \times \mathcal{Y} \mid \rho_{p}\left(\mathbf{x}_{0}, \ldots, \mathbf{x}_{p}\right)>\varepsilon\right\}, \quad \text { and } \\
& C=\left\{\left(\mathbf{x}_{0}, \ldots, \mathbf{x}_{p}, \mathbf{y}\right) \in \Omega \times \mathcal{Y} \mid\left(\mathbf{x}_{0}, \ldots, \mathbf{x}_{p}\right) \notin \mathcal{X}\right\} .
\end{aligned}
$$

We have $\Omega \backslash D=\Pi(\mathcal{E})$. In order to use the estimates on the Betti numbers of subPfaffian sets appearing in Corollary 2.21, we need to prove that the restriction of $\Pi$ to $\mathcal{E}$ is locally split. In order to do so, we introduce a shrinkage $\widetilde{C}$ of $C$ defined by

$$
\widetilde{C}=\left\{\left(\mathbf{x}_{0}, \ldots, \mathbf{x}_{p}, \mathbf{y}\right) \in \Omega \times \mathcal{Y} \mid\left(\mathbf{x}_{0}, \ldots, \mathbf{x}_{p}\right) \notin \overline{\mathcal{X}}\right\} .
$$


The point $\left(\mathbf{x}_{0}, \ldots, \mathbf{x}_{p}, \mathbf{y}\right)$ is in $C \backslash \widetilde{C}$ if and only if $\left(\mathbf{x}_{0}, \ldots, \mathbf{x}_{p}\right) \in \partial \mathcal{X}$, where

$$
\partial \mathcal{X}=\bigcup_{i=0}^{p}\left\{\left(\mathbf{x}_{0}, \ldots, \mathbf{x}_{p}\right) \in \Omega \mid \mathbf{x}_{i} \in \partial\left(X_{\lambda}\right)\right\} .
$$

Since $(X, Y)$ is a semi-Pfaffian couple, we know that $\partial\left(X_{\lambda}\right) \subseteq Y_{\lambda}$ (see Definition 2.10), so $\left(\mathbf{x}_{0}, \ldots, \mathbf{x}_{p}\right) \in \partial \mathcal{X}$ if and only if there exists $0 \leq i \leq p$ such that $\operatorname{dist}\left(\mathbf{x}_{i}, Y_{\lambda}\right)=0$. In particular, this means that $\Pi(C \backslash \widetilde{C})=\partial \mathcal{X} \subseteq \Pi(A)$, giving

$$
\Pi(A \cup B \cup C)=\Pi(A \cup B \cup \widetilde{C}) .
$$

Let $\widetilde{\mathcal{E}}=A \cup B \cup \widetilde{C}$. It is clear from the definition that $A, B$ and $\widetilde{C}$ are open subsets of $\Omega \times \mathcal{Y}$, so $\widetilde{\mathcal{E}}$ is open too, and according to Lemma 2.17 , the restriction of $\Pi$ to $\widetilde{\mathcal{E}}$ is locally split. But the restriction of $\Pi$ to $\mathcal{E}$ must be locally split too: the local sections of $\left.\Pi\right|_{\tilde{\mathcal{E}}}$ are local sections for $\left.\Pi\right|_{\mathcal{E}}$, and since $\Pi(\mathcal{E})=\Pi(\widetilde{\mathcal{E}})$, it is not necessary to check for the existence of other sections. ${ }^{3}$

If the format of $(X, Y)$ is bounded by $(n, \ell, \alpha, \beta, s)$, the format of $\mathcal{E}$ is bounded by

$$
(n(p+2),(p+2) \ell, \alpha, \max (2, \beta),(s+1)(p+2)) .
$$

Since $\mathcal{E}$ is semi-Pfaffian and $\left.\Pi\right|_{\mathcal{E}}$ is locally split, the Betti numbers of $\Pi(\mathcal{E})=\Omega \backslash D$ are bounded according to Corollary 2.21, and (21) follows.

In order to prove our main theorem, we will also need to relate the Betti numbers of the set $\left(\mathcal{U}_{\lambda}\right)^{p+1} \backslash D_{\lambda}^{p}(\varepsilon)$ to those of the complement of $D_{\lambda}^{p}(\varepsilon)$. This is achieved with the following lemma.

Lemma 4.3 Let $D$ and $\Omega$ be subsets of $\mathbb{R}^{N}$ such that $\bar{D} \subseteq \operatorname{int}(\Omega)$. Then, for all $q$, we have

$$
b_{q}\left(\mathbb{R}^{N} \backslash D\right) \leq b_{q}(\Omega \backslash D)
$$

Proof To prove the result, it is enough to show that the map $k: H_{q}(\Omega \backslash X) \rightarrow$ $H_{q}\left(\mathbb{R}^{N} \backslash X\right)$ induced by inclusion is surjective. Let us consider the following commutative diagram, where the rows are the exact sequences associated to the couples $\left(\mathbb{R}^{N} \backslash D, \Omega \backslash D\right)$ and $\left(\mathbb{R}^{N}, \Omega\right)$, respectively, and the vertical arrows are induced by the corresponding inclusions.

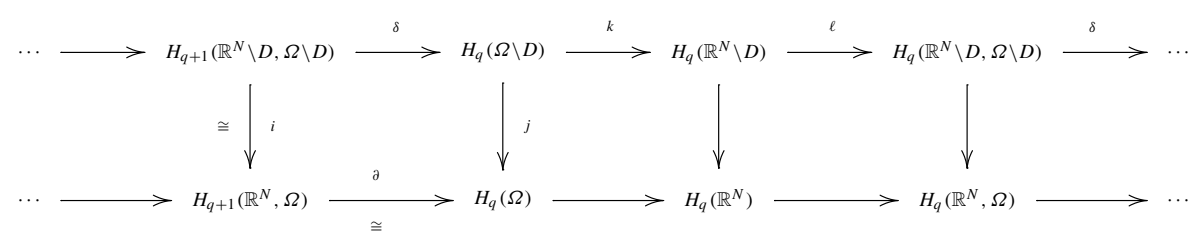

\footnotetext{
${ }^{3}$ For our argument, it is not enough to note that $\left.\Pi\right|_{\widetilde{\mathcal{E}}}$ is locally split and to apply the spectral sequence in that case: indeed, both $\mathcal{E}$ and $\widetilde{\mathcal{E}}$ are semi-Pfaffian, but the bound on the format of $\widetilde{\mathcal{E}}$ is much worse than the bound on the format of $\mathcal{E}$.
} 
Since $\bar{D} \subseteq \operatorname{int}(\Omega)$, the excision axiom asserts that the inclusion $i:\left(\mathbb{R}^{N} \backslash D, \Omega \backslash D\right) \hookrightarrow$ $\left(\mathbb{R}^{N}, \Omega\right)$ is an isomorphism on the homology level. Since $\mathbb{R}^{N}$ is contractible, the boundary maps $\partial$ in the exact sequence of the couple $\left(\mathbb{R}^{N}, \Omega\right)$ are isomorphisms; thus, we obtain that the composition $\partial \circ i: H_{q+1}\left(\mathbb{R}^{N} \backslash D, \Omega \backslash D\right) \rightarrow H_{q}(\Omega)$ is an isomorphism, and since this map is equal to $j \circ \delta$, the map $\delta$ must be injective.

By exactness of the first row at $H_{q}\left(\mathbb{R}^{N} \backslash D, \Omega \backslash D\right)$, we have im $\ell=\operatorname{ker} \delta=0$ (since $\delta$ injective), but by exactness at $H_{q}\left(\mathbb{R}^{N} \backslash D\right)$, we obtain $\operatorname{ker} \ell=H_{q}\left(\mathbb{R}^{N} \backslash D\right)=\operatorname{im} k$, and thus $k$ is surjective.

\subsection{Betti Numbers of a Relative Closure}

Our main result can now be obtained by combining the reduction of Sect. 4.1 and the estimates of Sect. 4.2.

Theorem 4.4 Let $(X, Y)$ be a semi-Pfaffian family defined in a domain $\mathcal{U} \subseteq \mathbb{R}^{n} \times \mathbb{R}_{+}$. If the format of $(X, Y)$ is bounded by $(n, \ell, \alpha, \beta, s)$, then for any integer $k \geq 1$, the Betti number $b_{k}\left((X, Y)_{0}\right)$ is bounded by

$$
2^{O\left(n^{2} k^{4} \ell^{2}\right)}[s k]^{O(k n)}[n k(\alpha+\beta)]^{O(k(n+\ell))},
$$

where the constants depend only on the domain $\mathcal{U}$.

Proof We established from Proposition 4.1 and Theorem 2.22 the bound

$$
b_{k}\left((X, Y)_{0}\right)=b_{k}\left(K_{0}\right) \leq \sum_{p+q=k} b_{q}\left(D_{\lambda}^{p}(\varepsilon)\right)
$$

for some suitable values $\lambda>0$ and $\varepsilon>0$.

Fix a value for $p$. We denote by $N=(p+1) n$ the dimension of the ambient space containing $D=D_{\lambda}^{p}(\varepsilon)$, and we use the notations introduced in the proof of Proposition 4.2. Since $D$ is compact, Alexander duality (Theorem 6.2.16 in [21]) gives $H^{q}(D) \cong \tilde{H}_{N-q-1}\left(\mathbb{R}^{N} \backslash D\right)$. Since $\Omega$ is open and $D$ is closed, Lemma 4.3 yields

$$
b_{q}(D) \leq b_{N-q-1}\left(\mathbb{R}^{N} \backslash D\right) \leq b_{N-q-1}(\Omega \backslash D) .
$$

According to Proposition 4.2, and since $p+q=k$, the Betti number $b_{N-q-1}(\Omega \backslash D)$ is bounded by

$$
2^{O\left(n^{2} p^{4} \ell^{2}\right)}[s(p+1)]^{O(k n)}[n k(\alpha+\beta)]^{O(k(n+\ell))} .
$$

Thus, $b_{q}\left(D_{\lambda}^{p}(\varepsilon)\right)$ is bounded by (24) too, and the sum $\sum_{p+q=k} b_{q}\left(D_{\lambda}^{p}(\varepsilon)\right)$ is bounded by (22), which proves the theorem.

Acknowledgements The author is indebted to Andrei Gabrielov for help with Lemma 4.3, and to Nicolai Vorobjov for useful comments on an earlier version of this paper.

를 Springer 


\section{References}

1. Basu, S.: On bounding the Betti numbers and computing the Euler characteristic of semi-algebraic sets. Discrete Comput. Geom. 22, 1-18 (1999)

2. Basu, S., Zell, T.: On projections of semi-algebraic sets defined by few quadratic inequalities. Arxiv:AG/0602398

3. Bochnak, J., Coste, M., Roy, M.-F.: Géométrie Algébrique Réelle. Springer, Berlin (1987). Revised 2nd edition in English: Springer (1998)

4. Coste, M.: An introduction to o-minimal geometry. Dip. Mat. Univ. Pisa, Dottorato di Ricerca in Matematica, Istituti Editoriali e Poligrafici Internazionali, 2000. Also available on http://www. ihp-raag.org/

5. Deligne, P.: Théorie de Hodge III. Publ. Math. IHES 44, 5-77 (1974)

6. Dugger, D., Isaksen, D.: Topological hypercovers and $\mathbb{A}^{1}$-realizations. Math. Z. 246, 667-689 (2004)

7. Gabrielov, A.: Frontier and closure of a semi-Pfaffian set. Discrete Comput. Geom. 19, 605-617 (1998)

8. Gabrielov, A.: Relative closure and the complexity of Pfaffian elimination. In: Discrete and Computational Geometry: The Goodman-Pollack Festschrift. Algorithms and Combinatorics, vol. 25. Springer, Berlin (2003)

9. Gabrielov, A.: Counter-examples to quantifier elimination for fewnomial and exponential expressions, Preprint (July 2006)

10. Gabrielov, A., Vorobjov, N.: Complexity of stratifications of semi-Pfaffian sets. Discrete Comput. Geom. 14, 71-91 (1995)

11. Gabrielov, A., Vorobjov, N.: Complexity of computations with Pfaffian and Noetherian functions. In: Ilyashenko, Y., Rousseau, C. (eds.) Normal Forms, Bifurcations and Finiteness Problems in Differential Equations. NATO Science Series II, vol. 137. Kluwer Academic, Dordrecht (2004)

12. Gabrielov, A., Vorobjov, N.: Betti numbers for quantifier-free formulae. Discrete Comput. Geom. 33, 395-401 (2005)

13. Gabrielov, A., Zell, T.: On the number of connected components of the relative closure of a semiPfaffian family. In: Algorithmic and Quantitative Real Algebraic Geometry. DIMACS: Series in Discrete Mathematics and Theoretical Computer Science, vol. 60. American Mathematical Society, Providence (2003)

14. Gabrielov, A., Vorobjov, N., Zell, T.: Betti numbers of semialgebraic and sub-Pfaffian sets. J. Lond. Math. Soc. 69, 27-43 (2004)

15. Karpinski, M., Macintyre, A.: A generalization of Wilkie's theorem of the complement, and an application to Pfaffian closure. Selecta Math. (N.S.) 5, 507-516 (1999)

16. Khovanskii, A.G.: Fewnomials. American Mathematical Society, Providence (1991)

17. Lion, J.-M., Rolin, J.-P.: Volumes, Feuilles de Rolle de Feuilletages analytiques et Théorème de Wilkie. Ann. Fac. Sci. Toulouse Math. 7, 93-112 (1998)

18. Lion, J.-M., Speissegger, P.: The theorem of the complement for sub-Pfaffian sets. Preprint. Available at http://www.math.mcmaster.ca/ speisseg/

19. Montaña, J.L., Morais, J.E., Pardo, L.M.: Lower bounds for arithmetic networks II: Sum of Betti numbers. Appl. Algebra Eng. Commun. Comput. 7, 41-51 (1996)

20. Osgood, W.: On functions of several complex variables. Trans. Am. Math. Soc. 17(1), 1-8 (1916)

21. Spanier, E.: Algebraic Topology. Springer, New York (1981)

22. Speissegger, P.: The Pfaffian closure of an o-minimal structure. J. Reine Angew. Math. 508, 189-211 (1999)

23. van den Dries, L.: Tame Topology and o-Minimal Structures. LMS Lecture Note Series, vol. 248. Cambridge University Press, Cambridge (1998)

24. Wilkie, A.J.: A theorem of the complement and some new o-minimal structures. Selecta Math. (N.S.) 5, 397-421 (1999)

25. Zell, T.: Betti numbers of semi-Pfaffian sets. J. Pure Appl. Algebra 139, 323-338 (1999). Effective methods in algebraic geometry (Saint-Malo, 1998)

26. Zell, T.: Topology of definable Hausdorff limits. Discrete Comput. Geom. 33, 423-443 (2005) 